\title{
Assembly of eight spherical magnets into a dotriacontapole configuration
}

\author{
Stefan Hartung,,${ }^{1, *}$ Felix Sommer, ${ }^{1}$ Simeon Völkel,,${ }^{1}$ Johannes Schönke, ${ }^{2, \dagger}$ and Ingo Rehberg ${ }^{1, \ddagger}$ \\ ${ }^{1}$ Experimentalphysik V, Universität Bayreuth, D-95440 Bayreuth, Germany \\ ${ }^{2}$ Okinawa Institute of Science and Technology Graduate University, Onna, Okinawa 904-0495, Japan
}

(Received 30 August 2018; revised manuscript received 31 October 2018; published 13 December 2018)

\begin{abstract}
The magnetic field of a cuboidal cluster of eight magnetic spheres is measured. It decays with the inverse seventh power of the distance. This corresponds formally to a multipole named a dotriacontapole. This strong decay is explained on the basis of dipole-dipole interaction and the symmetry of the ensuing ground state of the cuboidal cluster. A method to build such dotriacontapoles is provided.
\end{abstract}

DOI: 10.1103/PhysRevB.98.214424

\section{INTRODUCTION}

Within the forces determining the interplay of condensed matter, the dipole-dipole interaction can be considered as the most important one, because monopoles do not exist for neutral matter, and pure quadrupole, octopole, or hexadecapol interaction tends to be masked by induced dipole moments. While the interaction of quadrupoles is not too exotic [1] and includes examples from continuum mechanics [2], pure octopole or even higher order interaction is different. Here we demonstrate that the combination of eight dipoles in a simple cubic arrangement leads to a 32-pole or dotriacontapole.

The exploration of the cuboidal dipole arrangement discussed here is triggered by the investigation of magnetic nanoparticles, which have been reported to self-assemble into such configurations [3,4]. The most elementary cluster of this type contains only eight particles. It can also be assembled macroscopically as a cubic cluster from eight magnetic spheres, as indicated by the left-hand side inset of Fig. 1, and described previously $[5,6]$. The ground state of this arrangement is stable, and an interesting continuum [5,7]. In this state, the spheres attract each other by the magnetic interaction. The cuboidal arrangement is an attractor, provided that the spheres are brought sufficiently close to that configuration and are allowed to adjust their orientation towards the ground state, i.e., their mutual friction must not be too large. That is the reason why the arrangement shown by the left-hand side inset of Fig. 1 can be assembled without needing a tremendous amount of dexterity, and in that sense the arrangement can be considered as almost self-assembled.

While the hallmark of a dipole is its field decay with the third power of the distance, the combination of eight dipoles could be expected to form a 16-pole or hexadecapole with a decay according to the sixth power. Amazingly enough, it turns out that the ground state of a cuboidal cluster of eight dipoles shows a field decay with the seventh power. This is

\footnotetext{
*Stefan.Hartung@uni-bayreuth.de

†Johannes.Schoenke@ oist.jp

${ }^{\ddagger}$ Ingo.Rehberg@uni-bayreuth.de
}

explained by the symmetry of the ensuing ground state which make all lower-order terms vanish.

\section{EXPERIMENTAL RESULTS}

For reaching the ground state of the cluster, the eight spheres should be allowed to rotate freely. For that purpose it is useful to provide a Teflon ${ }^{\circledR}$ spacer to reduce the friction of the spheres, as shown in the right-hand side inset of Fig. 1. Here, the eight neodymium magnets of diameter $d=(19 \pm$ $0.05) \mathrm{mm}$ are arranged in a cuboidal configuration by the holes at the corners of the white Teflon ${ }^{\circledR}$ cube, and kept at an edge length $L=(39.5 \pm 0.05) \mathrm{mm}$ by means of the nonmagnetic Teflon ${ }^{\circledR}$ spacer. A hole is drilled into that spacer along the face diagonal, the $(1,1,0)$ direction of the cube. This allows us to move the Hall probe (the black tip) into the cuboid, down to its center, by means of a stepper motor, using $0.1-\mathrm{mm}$ steps. We adjust the spheres within their continuous ground state to maximize the measured magnetic-flux density. This is achieved by manually turning just one sphere around the space diagonal as rotation axis; the other ones follow accordingly due to the magnetic interaction.

The measured magnetic-flux density along the $(1,1,0)$ direction is shown in Fig. 1. It has a maximum at about $r=28 \mathrm{~mm}$ - where the Hall probe is closest to the spheresand decays to zero both when approaching the center, and when increasing the distance from the cube. The solid line corresponds to a fit of the numerical superposition of the flux densities of eight accordingly arranged point dipoles, as given by (1) discussed below.

The most important feature of this cuboidal arrangement of dipoles is the unusually steep decrease of the magneticflux density outside the cube. To quantify this decrease, Fig. 2 shows the data from Fig. 1 in a logarithmic plot. It becomes obvious that the magnetic-flux density decays with the inverse seventh power of the distance. To characterize this magnetic cluster with an appropriate name, it must be recalled that the field of dipoles decays with the third power, quadrupoles with the fourth power, and so on. In that sense, the seventh power corresponds to a dotriacontapole.

The fact that the field is expected to be zero at that center of the arrangement is caused by the symmetry of the 


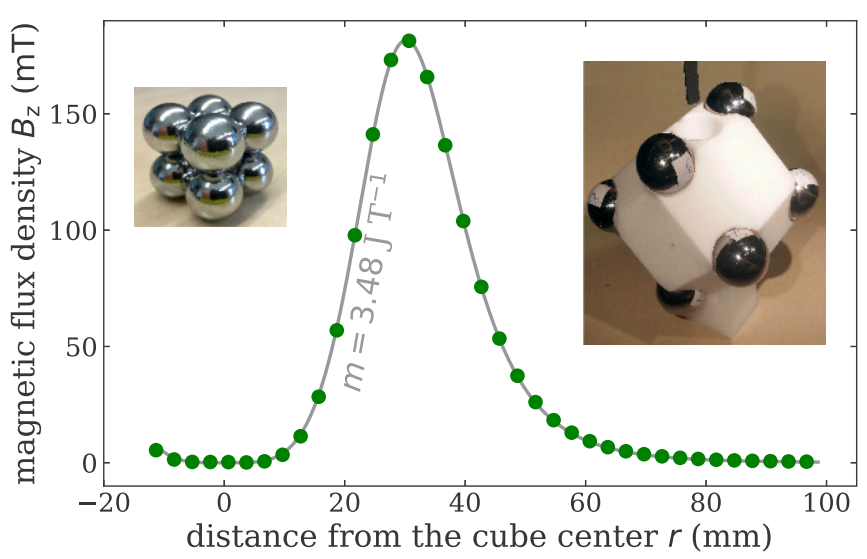

FIG. 1. Magnetic-flux density as measured along a straight path through the center of the cuboid. Only every 30 th data point is shown. The solid line corresponds to the numerical superposition of the flux densities of eight accordingly arranged point dipoles with a magnetic moment $m=3.48 \mathrm{~J} \mathrm{~T}^{-1}$. The left-hand side inset shows the principal cuboidal arrangement of the eight magnetic spheres, and the righthand side inset a geometrically similar arrangement, but here with a white Teflon ${ }^{\circledR}$ spacer. The hole in that spacer allows us to take data inside the cuboid by means of the Hall probe, which is visible as the black part above the hole.

ground state. The measured deviations from that value can be attributed to geometrical and experimental imperfections: The dipole moments are not mathematically identical; they might not have reached their ground state due to the finite amount of friction, and the Hall probe can reach the center of the arrangement only with a mm precision.

The increase of the flux density with the fourth power is in agreement with the numerical evaluation of the ground state. According to that simulation, it even seems to be universal, i.e., independent of the direction along which the field is calculated. Compared to the seventh power of the decay this fourth power seems less exotic. It is somehow reminiscent of the field near the center of a Helmholtz pair of coils, where

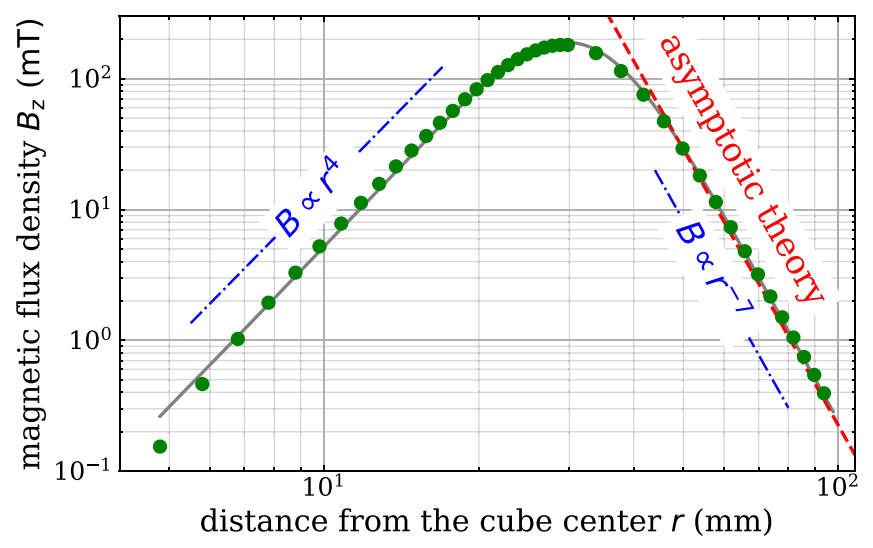

FIG. 2. The magnetic-flux density measured along a path starting from the center of the cuboid is represented by the circles. Only every tenth data point is shown at the left-hand side of the maximum, and every 40th data point at the right-hand side. The solid line is the same numerically obtained curve as in Fig. 1. The dash-dotted lines are for comparison with the expected asymptotic slopes. The dashed line depicts the analytical solution (7) for the far field.

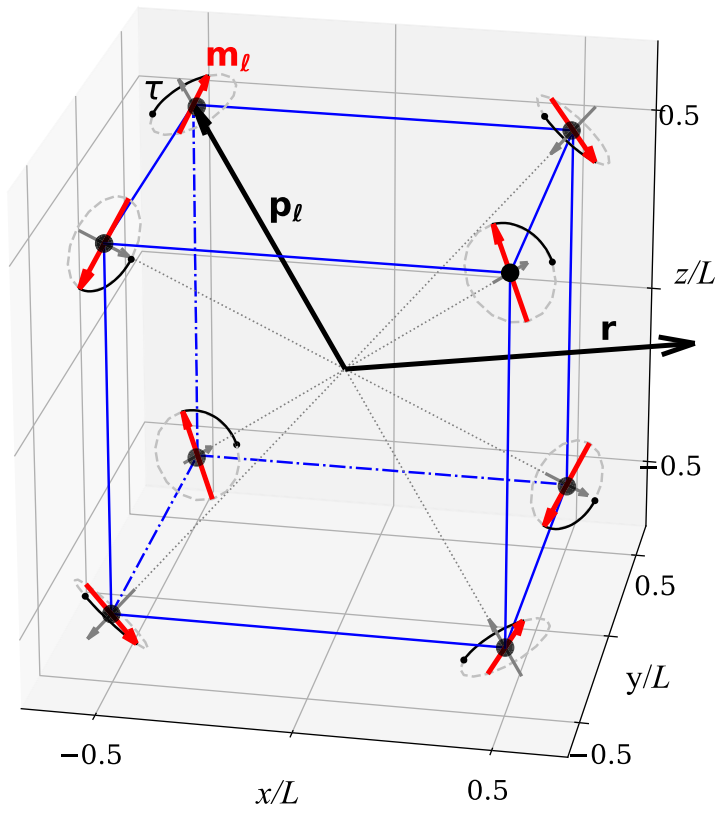

FIG. 3. The position $\mathbf{r}$ and the position vectors $\mathbf{p}_{\ell}$ of the dipole moments $\mathbf{m}_{\ell}$ are taken from the center of the cluster. The orientations of the dipoles in the continuous ground state are determined by the angle $\tau$. The dipole configuration is sketched here for $\tau=90^{\circ}$, which corresponds to the largest negative value of $B_{\mathrm{z}}$ along the $(1,1,0)$ direction.

the second-order terms vanish at a singular value of the coil distance.

\section{THEORY}

The scalar potential $\phi$ at position $\mathbf{r}$ for a distribution of $N$ dipoles with position vectors $\mathbf{p}_{\ell}$ and dipole moments $\mathbf{m}_{\ell}$ (see Fig. 3) is given by

$$
\phi=\sum_{\ell=1}^{N} \frac{\mathbf{m}_{\ell} \cdot\left(\mathbf{r}-\mathbf{p}_{\ell}\right)}{4 \pi\left|\mathbf{r}-\mathbf{p}_{\ell}\right|^{3}} .
$$

This equation is expected to describe the cluster field, because the individual spheres have a pure dipole field, in agreement with the theoretical expectation for homogeneously magnetized spheres [8] and our measurements presented in Appendix $A$. The numerical results of this equation correspond to the gray lines in Figs. 1 and 2.

To explain the behavior of the magnetic-flux density $\mathbf{B}$ in the far field, we perform a multidipole expansion, where the potential is expanded in a series for $\left|\mathbf{p}_{\ell}\right| \ll|\mathbf{r}|$,

$$
\phi=\left.\sum_{\nu=0}^{\infty} \frac{1}{\nu !} \sum_{\ell=1}^{N} \frac{\partial^{v} \phi}{\partial \mathbf{p}_{\ell}^{v}}\right|_{\mathbf{p}_{\ell}=0} \cdot(\underbrace{\left(\mathbf{p}_{\ell} \otimes \cdots \otimes \mathbf{p}_{\ell}\right.}_{\nu \text { times }}) .
$$

As an example, the quadrupole (second term in the expansion) reads

$$
\begin{aligned}
\phi^{(2)} & =\frac{1}{4 \pi|\mathbf{r}|^{5}} \sum_{\ell=1}^{N}\left[3\left(\mathbf{m}_{\ell} \cdot \mathbf{r}\right) \mathbf{r}-|\mathbf{r}|^{2} \mathbf{m}_{\ell}\right] \cdot \mathbf{p}_{\ell} \\
& =\frac{1}{4 \pi|\mathbf{r}|^{5}} \sum_{\ell=1}^{N}[\underbrace{3 \mathbf{m}_{\ell} \otimes \mathbf{p}_{\ell}-\left(\mathbf{m}_{\ell} \cdot \mathbf{p}_{\ell}\right) \mathbf{I}}_{\mathcal{M}^{2}}] \cdot(\mathbf{r} \otimes \mathbf{r}),
\end{aligned}
$$


with the second-order unit tensor I. The second-order tensor $\mathcal{M}^{2}$ is the quadrupole moment. Using Cartesian coordinates $\mathbf{p}_{\ell}=\left(p_{\ell}^{x}, p_{\ell}^{y}, p_{\ell}^{z}\right), \mathbf{m}_{\ell}=\left(m_{\ell}^{x}, m_{\ell}^{y}, m_{\ell}^{z}\right)$ and $\mathbf{r}=(x, y, z)$, we obtain

$$
\begin{aligned}
\phi^{(2)}= & \frac{1}{4 \pi|\mathbf{r}|^{5}}[\underbrace{\sum_{\ell=1}^{N}\left(2 p_{\ell}^{x} m_{\ell}^{x}-p_{\ell}^{y} m_{\ell}^{y}-p_{\ell}^{z} m_{\ell}^{z}\right)}_{\mathcal{M}_{200}^{2}} x^{2} \\
& +\underbrace{3 \sum_{\ell=1}^{N}\left(p_{\ell}^{x} m_{\ell}^{y}+p_{\ell}^{y} m_{\ell}^{x}\right)}_{\mathcal{M}_{110}^{2}} x y+\cdots] .
\end{aligned}
$$

$\mathcal{M}_{i j k}^{2}$ are the Cartesian components of the moment $\mathcal{M}^{2}$ with $i+j+k=2$. Using the moments, the potential can be written as

$$
\phi=\sum_{\alpha=1}^{\infty} \phi^{(\alpha)}=\sum_{\alpha=1}^{\infty} \frac{1}{4 \pi|\mathbf{r}|^{2 \alpha+1}} \sum_{i+j+k=\alpha} \mathcal{M}_{i j k}^{\alpha} x^{i} y^{j} z^{k} .
$$

The cube ground state [5,9] is a highly shielded structure. For a cube with edge length $L$ and dipole moment magnitudes $\left|\mathbf{m}_{\ell}\right|=m$ we have

$$
\begin{aligned}
& \text { (dipole) } \mathcal{M}_{i j k}^{1}=0, \\
& \text { (quadrupole) } \mathcal{M}_{i j k}^{2}=0, \\
& \text { (octopole) } \mathcal{M}_{i j k}^{3}=0, \\
& \text { (hexadecapole) } \mathcal{M}_{i j k}^{4}=0, \\
& \text { (dotriacontapole) } \mathcal{M}_{311}^{5}=C \sin (\tau+\pi / 3), \\
& \mathcal{M}_{131}^{5}=C \sin (\tau+5 \pi / 3), \\
& \mathcal{M}_{113}^{5}=C \sin (\tau+9 \pi / 3),
\end{aligned}
$$

where $\tau=0 \ldots 2 \pi$ is the current phase angle [5,9] of the continuous ground state as indicated in Fig. 3 and Appendix B, and $C=105 \sqrt{3 / 2} L^{4} m$. There are restrictions for the cube moments following from the symmetries of the ground state [5]. The potential $\phi$ has to be zero in the three planes, $x=0, y=0, z=0$, as well as on the four volume diagonals, $|x|=|y|=|z|$. Together with (2) this leads to conditions for the nonzero moments $\mathcal{M}_{i j k}^{\alpha}$ :

$$
i, j, k \text { positive, odd } \Rightarrow \alpha \text { odd, and } \sum_{i+j+k=\alpha} \mathcal{M}_{i j k}^{\alpha}=0 .
$$

This explains why the first nonzero moments appear in the dotriacontapole,

$$
\phi^{(5)}=\frac{\mathcal{M}_{311}^{5} x^{3} y z+\mathcal{M}_{131}^{5} x y^{3} z+\mathcal{M}_{113}^{5} x y z^{3}}{4 \pi|\mathbf{r}|^{11}} .
$$

The magnetic-flux density is related to the potential through $\mathbf{B}=-\mu_{0} \partial \phi / \partial \mathbf{r}$. We parametrize the measurement along the direction $(1,1,0)$ with the radius parameter $s$ through $(x, y, z)=(s, s, 0) / \sqrt{2}$ and obtain the following expression for the $z$ component of the magnetic-flux density from (3)-(6):

$$
\begin{aligned}
B_{z}(s, \tau) & =-\left.\mu_{0} \frac{\partial \phi}{\partial z}\right|_{x=y=s / \sqrt{2}, z=0} \\
& =-\frac{105 \sqrt{3 / 2} \mu_{0} L^{4} m \sin \tau}{16 \pi s^{7}}+\mathcal{O}\left(\frac{1}{s^{9}}\right) .
\end{aligned}
$$

The next order decays with $|\mathbf{B}| \propto 1 / s^{9}$ because all moments with even $\alpha$ are zero.

Equation (7) is displayed in Fig. 2 by the dashed line. The solid lines in Figs. 1 and 2 are obtained numerically from the exact (1), with $\tau=90^{\circ}$ taken as the phase angle of the continuous ground state (see Appendix C). It is amazing that this asymptotic prediction reaches the exact solution already at a distance of about $50 \mathrm{~mm}$, which can be considered as sheer luck from an experimental point of view, because the field is hardly detectable for our equipment at distances larger than $100 \mathrm{~mm}$. For the measurements shown there, the angle $\tau$ was adjusted manually to obtain the largest signal of the Hall probe to achieve an optimal signal-to-noise ratio. This corresponds either to $\tau=90^{\circ}$ or to $\tau=270^{\circ}$.

Note that the shape of the $B(r)$ curve shown in Figs. 1 and 2 is not universal, it rather depends on the direction of the line along which the flux density is measured. The $1 / r^{7}$ decay, however, is a universal feature for all directions in the far-field limit, $\left|\mathbf{p}_{\ell}\right| \ll|\mathbf{r}|$.

\section{CONCLUSION AND OUTLOOK}

In summary, we have demonstrated that eight spherical permanent magnets assemble into a configuration which behaves like a dotriacontapole. This can be explained by a model based on pure dipole-dipole interaction. This model is based on symmetry considerations which are an idealization of the experimental situation. The measurements make it clear that the conclusions drawn from the idealization are robust against (small) distortions, in particular the decay of the magneticflux density with $1 / r^{7}-$ a hallmark for a highly shielded structure-survives.

This finding implies that storing strong magnets in a cubic packing might be the optimal way for suppressing their field in the outer surrounding. Moreover, the extremely steep field decay has remarkable consequences for the clustering dynamics: If two dipole spheres, initially separated by say ten diameters, needed one second to collide due to their attractive force, for dotriacontapoles of comparable strength, this process would take more than one year (see Appendix B). Thus, dipoles which manage to arrange themselves in this configuration are fairly robust against further clustering. This argument is scale invariant. It applies to macroscopic granules in the early stages of planet formation [10], but could also shed some light on the self-assembly dynamics of colloidal nanomagnets [3,4] used for medical applications [11].

The plastic spheres shown in Fig. 4 demonstrate an attempt to build a handful of such dotriacontapoles with the help of a three-dimensional (3D) printer. Each sphere contains eight magnetic dipoles in a cubic arrangement. This is provided by three perpendicular walls inside these spheres, indicated in the left-hand side inset, and eight holes along the space 


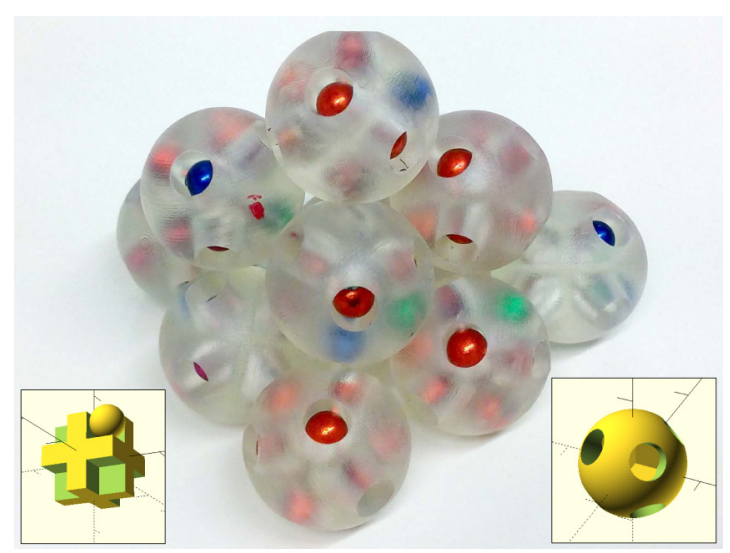

FIG. 4. A cluster of 3D-printed dotriacontapoles. The inner part of these spheres contains three perpendicular walls as indicated by the left-hand side inset. The colored magnetic spheres of $5 \mathrm{~mm}$ diameter are placed inside these plastic spheres by the eight holes along the space diagonals, as indicated by the right-hand side inset.

diagonals, as indicated by the right-hand side inset. These plastic spheres should thus interact with an extremely shortranged interaction force, which should asymptotically decay with the inverse 12th power of the mutual distanceprovided that the magnetic dipoles inside a sphere are in their ground state. Measuring such a short-range interaction between dipole clusters provides a challenge left to be faced in future work.

\section{ACKNOWLEDGMENTS}

It is a pleasure to thank F. Braun, K. Huang, R. Richter, W. Schöpf, and A. Weber for valuable hints and discussions. This work has been supported by the Deutsche Forschungsgemeinschaft (DFG) - Project No. 389197450.

\section{APPENDIX A: MAGNETIC SPHERES AS DIPOLES}

The magnetic spheres (MK-19-C from magnets4you $\mathrm{GmbH})$ have a diameter of $d=(19 \pm 0.05) \mathrm{mm}$. For explaining the experimental findings with a theoretical model based on pure dipole-dipole interaction, it is crucial to demonstrate that these spheres can be described as magnetically hard point dipoles. Thus, we have measured the axial component of the magnetic-flux density $B_{\mathrm{x}}$ of a single sphere along the $x$ direction in a $170-\mathrm{mm} \times 20-\mathrm{mm} x y$ plane, as shown in the inset of Fig. 5. The flux density is measured by a Hall probe (HU-ST1-184605, MAGNET-PHYSIK Dr. Steingroever $\mathrm{GmbH}$ ). The $3 \mathrm{D}$ positioning of this probe is done with a stepper motor (High-Z S-400T, with Zero-3 controller from CNC-STEP), the interface (CNCPod) is programmable in G-Code, DIN/ISO 66025. A single-board microcontroller (Leonardo, Arduino) is additionally used for interfacing it to a PC.

To emphasize deviations from the point dipole approximation, and to extract the underlying magnetic moment, we make use of the theoretically expected flux density of a point

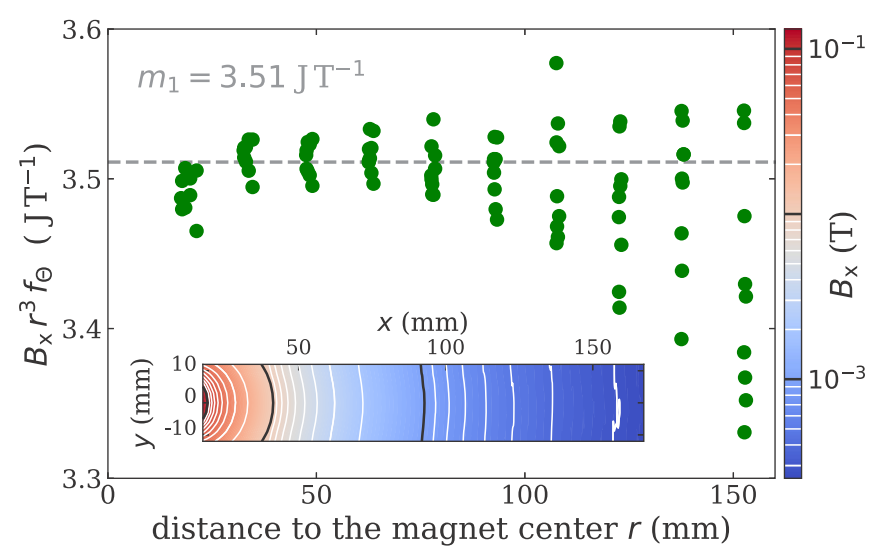

FIG. 5. The inset shows $B_{\mathrm{x}}(x, y)$ measured in a plane, color coded in red for strong fields to blue for weak ones. The direction of the $x$ coordinate is chosen to be parallel to the dipole moment $\mathbf{m}$ and forms a horizontal plane with the perpendicular coordinate $y$. An estimator for the magnetic moment is obtained from these data with (A2). The result is displayed as a function of $r$ by the circles. The dashed line represents the mean value $m_{1}$ of these data.

dipole [8],

$$
B_{\mathrm{x}}=\frac{\mu_{0}}{4 \pi} \frac{m\left(3 \cos ^{2} \Theta-1\right)}{r^{3}},
$$

with $\Theta=\arctan (y / x), r=\sqrt{x^{2}+y^{2}}$ measuring the angle between the dipole moment and the position vector, and the magnetic constant $\mu_{0}$. With the shorthand notation $\frac{4 \pi}{\mu_{0}\left(3 \cos ^{2} \Theta-1\right)}=f_{\Theta}$, this provides the magnitude of the magnetic moment,

$$
m=B_{\mathrm{x}} r^{3} f_{\Theta} .
$$

The resulting $m$ as a function of the measured value of $B_{\mathrm{x}}(x, y)$ is plotted in Fig. 5 as a function of the distance of the Hall probe from the center of the sphere. The increasing

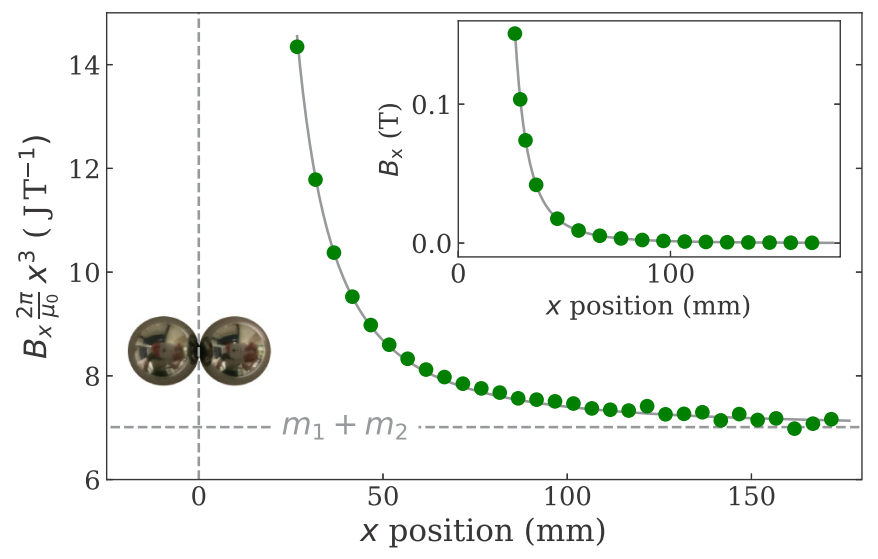

FIG. 6. Data obtained from the measurements of the magneticflux density $B_{x}$ of two spheres in contact. The raw data are shown in the inset, and the solid line shows the calculated superposition of two dipole fields. $B_{x}$ scaled with $x^{3} 2 \pi / \mu_{0}$ is shown in the larger plot. The horizontal dashed line represents the sum of the magnetic moments of the isolated spheres. The dashed vertical line represents the origin at the contact point of the spheres. 
scatter at larger distances $r$ is caused by the fast decay of the magnetic-flux density. Based on these data, it seems safe to conclude that the point dipole approximation for the magnetic-flux density of the sphere is reliable within $\pm 2 \%$. The mean value is $(3.51 \pm 0.18) \mathrm{J} \mathrm{T}^{-1}$, which is well within the $(3.54 \pm 0.11) \mathrm{J} \mathrm{T}^{-1}$ claimed by the manufacturer. We have measured all eight dipoles used in the experiments described here in a similar way, they differ by an amount of $\pm 3 \%$.

To measure the mutual influence of such magnetic spheres, we brought them in direct contact as shown in the left-hand side inset of Fig. 6. The measured flux density along the axis of the resulting two-dipole cluster is shown as the righthand side inset in Fig. 6. The position of the Hall probe is measured as the distance from the center between the spheres. The data reveal roughly the typical $1 / x^{3}$ descent of a dipole, but deviations from that scaling are hard to judge from this inset plot. To get a better resolution for the deviations from the overall $1 / x^{3}$ decay, the data were multiplied with $x^{3}$. After scaling with $2 \pi / \mu_{0}$ one gets an estimate for the magnetic moment, which is displayed on the vertical axis of Fig. 6. These scaled data decay monotonically with the position $x$ and reach the value of the sum of the two magnetic moments asymptotically, which is indicated by the dashed line. The solid line is the theoretical estimation, based on the superposition of the fields of the individually measured moments $m_{1}=3.51 \mathrm{~J} \mathrm{~T}^{-1}$ and $m_{2}=3.50 \mathrm{~J} \mathrm{~T}^{-1}$, with their mutual distance given by the diameter of the spheres. The good agreement between this curve and the data indicates that the magnets are hard ones: Their magnetic moment stays constant even under the influence of the immediately adjacent other magnet, at least within the experimental resolution on a percentage level.

\section{APPENDIX B: DIPOLE ORIENTATION IN THE CUBE GROUND STATE}

The dipole orientation within the ground state of the cuboidal cluster can be parametrized by a single parameter, namely the phase angle $\tau=0^{\circ} \ldots 360^{\circ}$ [5,9]. The configuration for the angle $\tau=90^{\circ}$ is illustrated in Fig. 3. The source code of a Python script animating this state together with the corresponding fields for adjustable values of $\tau$ is available [12]. The exact positions and orientations of the dipoles with respect to $\tau$ are provided by Table I.

\section{APPENDIX C: ASSEMBLY TIME FOR DIPOLES VERSUS THAT FOR DOTRIACONTAPOLES}

The time $T_{\mathrm{m}}$ for two multipoles of diameter $d$ starting at a distance of $10 d$ to come into contact under the influence of
TABLE I. Description of $\mathbf{p}_{i}$ and $\mathbf{m}_{i}$ in the cube ground state.

\begin{tabular}{|c|c|c|}
\hline$i$ & $\mathbf{p}_{i}$ & $\mathbf{m}_{i} \cdot \sqrt{3 / 2} / m$ \\
\hline & $(0.5)$ & $\left(-\sin \left(\tau-240^{\circ}\right)\right)$ \\
\hline \multirow[t]{2}{*}{1} & 0.5 & $-\sin \left(\tau-120^{\circ}\right)$ \\
\hline & $(0.5)$ & $-\sin (\tau)$ \\
\hline \multirow{3}{*}{2} & $(0.5)$ & $\left(+\sin \left(\tau-240^{\circ}\right)\right)$ \\
\hline & 0.5 & $+\sin \left(\tau-120^{\circ}\right)$ \\
\hline & $(-0.5)$ & $-\sin (\tau)$ \\
\hline \multirow{3}{*}{3} & $(0.5)$ & $\left(+\sin \left(\tau-240^{\circ}\right)\right)$ \\
\hline & -0.5 & $-\sin \left(\tau-120^{\circ}\right)$ \\
\hline & 0.5 & $+\sin (\tau)$ \\
\hline \multirow{3}{*}{4} & $(0.5)$ & $\left(-\sin \left(\tau-240^{\circ}\right)\right)$ \\
\hline & -0.5 & $+\sin \left(\tau-120^{\circ}\right)$ \\
\hline & -0.5 & $+\sin (\tau)$ \\
\hline \multirow{3}{*}{5} & $(-0.5)$ & $\left(-\sin \left(\tau-240^{\circ}\right)\right)$ \\
\hline & 0.5 & $+\sin \left(\tau-120^{\circ}\right)$ \\
\hline & 0.5 & $(+\sin (\tau)$ \\
\hline \multirow{3}{*}{6} & $(-0.5)$ & $\left(+\sin \left(\tau-240^{\circ}\right)\right)$ \\
\hline & 0.5 & $-\sin \left(\tau-120^{\circ}\right)$ \\
\hline & -0.5 & $+\sin (\tau)$ \\
\hline \multirow{3}{*}{7} & $(-0.5)$ & $\left(+\sin \left(\tau-240^{\circ}\right)\right)$ \\
\hline & -0.5 & $+\sin \left(\tau-120^{\circ}\right)$ \\
\hline & $(0.5)$ & $(-\sin (\tau)$ \\
\hline \multirow{3}{*}{8} & $(-0.5)$ & $\left(-\sin \left(\tau-240^{\circ}\right)\right)$ \\
\hline & -0.5 & $-\sin \left(\tau-120^{\circ}\right)$ \\
\hline & -0.5 & $-\sin (\tau)$ \\
\hline
\end{tabular}

their mutual attraction - a characteristic time for the dynamics of the self-assembly of magnetic clusters [3,4] -is obtained by integrating over their inverse velocity. When assuming that these particles are suspended in a viscous fluid, that velocity is proportional to the attractive force (Stokes's law). $T_{2}$ denotes the pair of dipoles, $T_{32}$ denotes the pair of dotriacontapoles. The attracting force of these multipole pairs is assumed to be the same when they are in contact at the distance of $1 d$,

$$
\begin{aligned}
\frac{T_{32}}{T_{2}} & =\frac{\int_{5 d}^{d / 2} \frac{1}{v_{32}} d r}{\int_{5 d}^{d / 2} \frac{1}{v_{2}} d r} \stackrel{v \propto F}{=} \frac{\int_{5 d}^{d / 2}-\left(\frac{2 r}{d}\right)^{12} d r}{\int_{5 d}^{d / 2}-\left(\frac{2 r}{d}\right)^{4} d r} \\
& =\frac{5}{13} \frac{10^{13}-1}{10^{5}-1} \approx 0.4 \times 10^{8} .
\end{aligned}
$$

This ratio turns, e.g., $1 s$ for a dipole pair into $1 a$ for the corresponding pair of dotriacontapoles: They are fairly robust against further clustering.
[1] A. D. Buckingham, Q. Rev., Chem. Soc. 13, 183 (1959).

[2] D. Stamou, C. Duschl, and D. Johannsmann, Phys. Rev. E 62, 5263 (2000).

[3] S. Mehdizadeh Taheri, M. Michaelis, T. Friedrich, B. Förster, M. Drechsler, F. M. Römer, P. Bösecke, T. Narayanan, B.
Weber, I. Rehberg, S. Rosenfeldt, and S. Förster, Proc. Natl. Acad. Sci. USA 112, 14484 (2015).

[4] S. Rosenfeldt, S. Förster, T. Friedrich, I. Rehberg, and B. Weber, in Novel Magnetic Nanostructures, Advanced Nanomaterials, edited by N. Domracheva, M. Caporali, and 
E. Rentschler (Elsevier, Amsterdam, Netherlands, 2018), pp. 165-189.

[5] J. Schönke, T. M. Schneider, and I. Rehberg, Phys. Rev. B 91, 020410 (2015).

[6] S. Borgers, S. Völkel, W. Schöpf, and I. Rehberg, Am. J. Phys. 86, 460 (2018).

[7] P. I. Belobrov, R. S. Gekht, and V. A. Ignatchenko, Zh. Eksp. Teor. Fiz. 84, 1097 (1983) [Sov. Phys. JETP 57, 636 (1983)].

[8] J. D. Jackson, Classical Electrodynamics, 3rd ed. (Wiley, New York, 1999).
[9] J. Schönke, An interactive gallery of the dipole cube, http://ecps.ds.mpg.de/people/cube_gallery/cube_gallery.html (2015).

[10] J. Blum, Adv. Phys. 55, 881 (2006).

[11] T. M. Buzug, J. Borgert, T. Knopp, S. Biederer, T. F. Sattel, M. Erbe, and K. Lüdtke-Buzug, Magnetic Nanoparticles (World Scientific, Singapore, 2010).

[12] I. Rehberg, Ground state of the dipole cube, http:// www.staff.uni-bayreuth.de/ bt180034/dotriacontapole.html (2018). 